\title{
Genetic versus Environmental Analysis of Reproductive Behavior of Female Twins
}

\author{
Shayesteh Jahanfar ${ }^{1 *}$, Munn Sann Lye ${ }^{2}$
}

${ }^{1}$ School of Health Sciences, Central Michigan University, Mount Pleasant, MI, USA
${ }^{2}$ Department of Community Health, University Tunku Abdul Rahman, UTAR Kampar Campus, Kuala Lumpur, MALAYSIA
${ }^{*}$ Corresponding Author: jahan2s@cmich.edu

Citation: Jahanfar, S. and Lye, M. S. (2021). Genetic versus Environmental Analysis of Reproductive Behavior of Female Twins. European Journal of Environment and Public Health, 5(1), em0051. https://doi.org/10.29333/ejeph/8365

\section{ARTICLE INFO}

Received: 11 Dec. 2019

Revised: 23 May 2020

Accepted: 2 Jun. 2020

\author{
ABSTRACT \\ Purpose: The study aims at investigating the relationship between environmental and hereditary causes of \\ reproductive behaviour, which would otherwise be difficult to study.
}

Materials and Methods: This cross-sectional descriptive study of the female reproductive health of twins investigated the heritability of qualitative and quantitative measured variables related to the reproductive behaviour of adult twins. Subjects included192 identical and non-identical twins, 15 years of age and above, who were living in urban areas of Malaysia.

Results: Basic genetic analysis for variables related to reproductive behaviour revealed that the age of first pregnancy, number of pregnancies and age of first marriage were under genetic influence. Proband wise concordance rate analysis showed higher similarity between identical twins in adopting recommended reproductive behaviour such as undergoing pap smears and using family planning techniques. The model-fitting analysis supported these findings.

Conclusion: We concluded that genetic variation exists in individual differences in the reproductive behaviour of young female adults.

Keywords: reproductive behaviour, twin, genetic versus environment, proband wise concordance

\section{INTRODUCTION}

While many epidemiological and clinical studies have tried to improve our knowledge regarding reproductive behaviour, few studies have been done on twins. Reproductive behaviour is associated with human development (Christensen et al., 2003; Houle D., 1992), infertility (Day et al., 2015; Rahmioglu et al., 2014) and certain psychological disorders (Mehta et al., 2016). Infertility and sub-infertility that constitute about 20 $30 \%$ of infertility cases remain unexplained, while over 48.5 million couples are infertile worldwide (Mehta et al., 2016). Age of marriage and age of the pregnancy, although considered issues related to social sciences, are closely linked with one's health.

Family and twin studies have long been used to find the genetic or environmental effect of a trait. Even though twins are the same age and usually grow up in the same household, their experiences can differ greatly. Identical twins share all the same hereditary material (DNA), whereas fraternal twins share only about half of the same DNA. Greater similarity for a trait among identical twins than the same trait among fraternal twins implies that the influence of genetic factors may predominate over that of environmental factors. Twin studies help us to understand the effect of genes and the environment on a specific measured variables. This study aimed to examine the relative contribution of genetic and environmental components pertaining to reproductive behavior including the age of first pregnancy, sex of children, number of pregnancies, deliveries, abortions, and number of children.

\section{MATERIALS AND METHODS}

This was a cross-sectional and comparative study. The study setting included all urban areas from Malaysian providences. Volunteer twins entered the study. The target population were adult female-female, and female from female-male twins, including both dizygotic (DZ) and monozygotic (MZ) twins. For the purposes of asking reproductive health questions, an adult was some 15 years of age and above. If one twin was not willing to participate, 
his/her cooperation was sought through the other twin. If he/she still did not want to participate, the data from the first twin were not analyzed. The histories of the Malaysian Twin Registries are described elsewhere (Jahanfar and Jaffar, 2013; Jahanfar, Lye and Krishnarajah, 2013).

This study was funded by the University of Kuala Lumpur. The Ethics committee at the National Malaysian Research Registry approved the study (\# 7379).

To recruit twins, a poster and a pamphlet was designed, inviting all twins to register. These materials were sent to all public and private hospitals, clinics, high schools, colleges and universities, ministries, public offices and shopping centers. A website was designed for the same purpose. A YouTube video clip was produced to explain the registry in three different languages (Chinese, Bahasa Malaya and Tamil) and invited twins to participate in the study. The information was also disseminated through mass media such as radio stations and newspapers. The information included the objectives of establishing a twin registry and the benefits and advantages of such a program (Jahanfar and Jaffar, 2013).

Twins contacted the two registries by calling or sending an SMS to phone numbers given in the advertisement, sending an e-mail to the prepared website, or faxing or mailing a letter provided in the pamphlet. Information from the participants was collected via phone calls. At the first stage, 800 phone calls were made. There is no record of how many successful contacts were made. From the phone calls made, only 192 individual twins were willing to cooperate fully. Refusal to cooperate was due to several reasons: language barriers, subjects finding the information to be sensitive, and choosing not to cooperate after consultation with their partner. There is no information on the percentage of each of these reasons.

In all cases, an information sheet explaining the study's objectives was read for the twins over the phone and their consent to participate in the study and consent to publish the study results were obtained verbally. Details of their co-twin were obtained; the co-twins were contacted through SMS, phone call, e-mail, or mail to register and further data were obtained from them through phone calls.

Two research assistants at each study setting were available during working hours to answer the phone calls and handle the interviews by phone or face to face in the case where the twins were referred to the registry office. One researcher was available during the weekends to fill in the questionnaire if the twins required. Each interview took about 15 to 20 minutes. Female interviewers interviewed the twins when possible, to increase the response rate. Telephone interviews were conducted whenever possible, and attempts were made to fill the questionnaire at the first contact. All the questionnaires were filled in by the interviewer.

A standard pilot-tested questionnaire was designed using brainstorming and literature review. The questionnaire was sent to five reproductive health specialists for content validity. The reproductive health questionnaire consisted of two major sections. The first section included descriptive sociodemographic data. The second section included information related to reproductive behavior.

Data collected were as follows: age, sex, education, occupation, marital status, race, weight and height (memory recall: if in doubt they were asked to measure their weight and height if possible; a second phone call was made to confirm the correct data on weight and height if necessary), birth weight (if subjects had difficulty recalling this, they were asked to ask their parents about birth weight; in some cases, the researchers called the parents), the status of living (shared or separated), and zygosity (subjects were asked if they are identical or not, whether their friends and relatives have difficulty recognizing them and if as a child they used to wear an identical mark). In a population-based sample of femalefemale adult twins, self-reported zygosity in both members of the twin pair agreed with assigned zygosity in about 95\% of pairs (Kendler et al., 1993). A study from the Garvan Institute in Australia has shown that comparing the subject's stated zygosity with DNA matching has over 99\% accuracy (Jahanfar, 2008).

Reproductive behavior was assessed by asking the following questions: age of first pregnancy, sex of children, number of pregnancies, deliveries, abortions, and number of children. All participating twins in this study were living together during childhood. This was verified by asking them, "were you living together during childhood?"

The sample size was calculated using MX script via simulation, as described in Neale (1997). The number of samples depends on the $\mathrm{h}^{2}$ (heritability index) and $\mathrm{C}^{2}$ (Common environment index) for each variable. For example, for a variable such as "age of marriage", the heritability is 0.4 . The sample size of 190 was considered adequate to reject the null hypothesis at a power level of 0.80 and $\alpha$ level of 0.05 . The sample comprised 50\% MZ and 50\% DZ pairs reared together.

Statistical analysis and modelling techniques are explained elsewhere (Jahanfar, Lye and Krishnarajah, 2013) but described shortly in three sections: proband-wise concordance rate, SPSS was used for descriptive analysis. Then, a more comprehensive genetic analysis was conducted using the $\mathrm{Mx}$ 2.0 software (Neale, 1997).

\section{Statistical Analysis}

Data analysis is explained in four sections: Classical Falconer's model approach, proband-wise concordance rate, genetic modelling for qualitative variables and model fitting analysis.

\section{Classical Falconer's Model Approach}

Using twin data, it was possible to estimate the variance components using Falconer's formula (1966). Basic twin studies adopt this formula for estimation of heritability $\left(h^{2}\right)$. This is an index of the relative contribution of genetic effects to the total phenotypic variance and it is calculated using this formula: $h^{2}=2(r M Z-r D Z)$ where $r$ is the intraclass correlation coefficient. Comparing resemblances between twins for traits is most frequently used for calculation of heritability. Identical twins are twice as genetically similar as non-identical ones and heritability is approximate twice the difference in correlation between $\mathrm{MZ}$ and $\mathrm{DZ}$ twins.

The effect of shared environment or $C$ can be calculated using $\mathrm{h}^{2}$ as it contributes to the similarity between twins due to the effect of shared environment they are raised in. The shared environment is estimated by MZ correlation deducted from heritability value $\mathrm{C}^{2}=\left(\mathrm{rMZ}-\mathrm{h}^{2}\right)$ or $\mathrm{C}^{2}=(2 \mathrm{rDZ}-\mathrm{rMZ})$. Not- 
Twin 1

\begin{tabular}{|c|c|c|c|}
\hline \multirow{3}{*}{ Twin 2} & & 0 & 1 \\
\hline & 0 & $a$ & $\mathrm{~b}$ \\
\hline & 1 & $\mathrm{c}$ & $\mathrm{d}$ \\
\hline
\end{tabular}

$0=$ symptoms absent; $1=$ symptoms present

Figure 1. Contingency table

shared environment or $\mathrm{E}^{2}$ is a reflection of the degree to which identical twins raised together are dissimilar, $\mathrm{E}^{2}=(1-\mathrm{rMZ})$ or $\mathrm{E}^{2}=1-(\mathrm{h} 2+\mathrm{C} 2)$.

\section{Proband-wise Concordance Rate for Qualitative Variables}

This is the probability that the co-twin of a proband twin will also have a condition, a symptom of a disease. Every affected individual is considered a proband. Numbers of probands whose co-twins are affected are then divided by the number of probands. For instance, if we set a contingency table as Figure 4, the proband-wise concordance rate is calculated using this formula: $2 d /(2 d+b+c)$. It is worth noting that the number of probands with an affected co-twin is twice the number of concordant affected pairs. The influence of genetic component from proband-ascertained twin pairs is usually based on a difference between $\mathrm{MZ}$ and $\mathrm{DZ}$ concordance rates. If $M Z$ to $D Z$ concordance ratio is 2 to 1 , it means that MZ twins are twice as likely to be affected if their co-twin is affected than DZ twins. This suggests that the effect of additive genetic influences is possible for the measured trait.

\section{Genetic Modelling for Qualitative Variables}

Correlation in liability for each type of twins, known as tetrachoric correlations, can be estimated by maximum likelihood via MX. Contingency tables for MZ and DZ for every single variable was entered as a Matrix into MX script retrieved from MX website and output inclusive of $\mathrm{p}$ values, and chisquared fit to model was retrieved as well as $95 \% \mathrm{CI}$, lower and higher limits.

Quantifying the genetic and environmental contribution to a binominal variable is feasible by assuming that there is a continuous underlying liability to disease (Falconer, 1989). This threshold of liability divides individuals into two categories: with or without the disease or condition. In a twin sample, correlation in liability among twins can be estimated from the frequencies of concordant and discordant pairs, and it is called tetrachoric correlation. The assumption behind this correlation is that the underlying liability is following a normal distribution or can be transformed in one. According to Neale (1992), structural equation modelling can then be applied to the MZ and DZ twins' correlations to provide estimates of the relative genetic and or environmental factors contribute to determining the trait. For each phenotype, there are genetic variance inclusive of additive component (A) and a dominant component (D) as well as environmental variance inclusive of common or shared environment $(\mathrm{C})$ and unique or non-shared environment (E). The genetic model can then be represented as a linear structural equation:

$\mathrm{P}=\mathrm{aAi}+\mathrm{dDi}+\mathrm{cCi}+\mathrm{eEi}$

$\mathrm{Vp}=\mathrm{a} 2+\mathrm{d} 2+\mathrm{c} 2+\mathrm{e} 2=1$

Where $\mathrm{P}$ is the phenotype of the ith individual, scaled as a deviation from zero. $\mathrm{Vp}$ is the total phenotype variance of the population, representing the sum of the individual components, a2 stands for additive genetic variance, $\mathrm{d} 2$ : dominant genetic variance, c2: common environmental variance and e2: unique environmental variance. $\mathrm{A}, \mathrm{D}, \mathrm{C}$ and $\mathrm{E}$ are considered as uncorrelated latent factors with mean of zero and variance of one while $\mathrm{a}, \mathrm{d}, \mathrm{c}$ and e are regression coefficients of the observed variable on the latent factors and they indicate the strength of the relationship between latent factors, and the phenotype. If we calculate the square of the regression coefficients and add them together $\left(V A=a^{2}, V D=d^{2}\right.$, $\mathrm{VC}=\mathrm{c}^{2}, \mathrm{VE}=\mathrm{e}^{2}$ ), the result would be equal to the total phenotypic variance, which is 1 for threshold traits (Snieder, 1997).

For MZ twins, the correlation between additive and dominant genetic factors between the twin 1 and twin 2 is one. For DZ twins, these values are 0.5 and 0.25 , respectively. Correlations are unity between common environmental factors and zero between unique environmental factors. The observed tetrachoric correlations can, therefore be represented as the sum of the following components, for $\mathrm{MZ}$ and DZ twins, respectively: $r M Z=a^{2}+c^{2}+e^{2}$, and $r D Z=1 / 2 a^{2}+$ $c^{2}+1 / 4 e^{2}$, where rMZ and $\mathrm{rDZ}$ are the tetrachoric correlations. These values can be estimated using MX script for calculating tetrachoric correlations found from MX script library (http://mxscripts.ctglab.nl/index.php?page=mx_news).

Contingency tables for MZ and DZ were entered in the appropriate place in which the cells contained the frequencies of pairs concordant and discordant for the presence or absence of a condition or disease (such as hirsutism) for both MZ and DZ twin pairs.

\section{Model Fitting for Qualitative Data}

Using MX, models were fitted to the matrices by the method of maximum likelihood and the relative significance of $\mathrm{A}, \mathrm{C}$, and D were tested by removing them sequentially in specific sub-models by hierarchic chi-squared tests; the chisquared -value of a sub-model was subtracted from that of the full model. The degrees of freedom (df.) for this test was equal to the difference between the $\mathrm{df}$ for the full model and the submodel. This was done using MX script for ACE and ADE model fitting qualitative data.

The reconstructed contingency data from each of these models were compared with that of the observed twin data (i.e., the original $2 \times 2$ tables) and chi-square-values, $p$-values were generated. A low value for chi-square and a high $\mathrm{p}$-value indicates that the model offers a good description of the data. Akaike's Information Criterion (AIC, $\chi-2 \mathrm{df}$ ) was also generated to evaluate the fit of the models. According to Neale (1992), a model with the lowest AIC reflects the best balance of goodness of fit and parsimony.

The objective of the model fitting method was to acquire the simplest model or the best fitting model that provides an adequate description of the data. The heritability $\left(h^{2}\right)$ for the 
Table 1. Description of quantitative variables of reproductive behavior for both MZ and DZ twins ( $\mathrm{n}=193)$

\begin{tabular}{ccccc}
\hline Variable & Mean & SD & Min & Max \\
\hline Age of marriage & 20.98 & 5.036 & 10 & 35 \\
\hline Age of first pregnancy & 21.17 & 4.603 & 11 & 32 \\
\hline Number of pregnancy & 2.9 & 2.03 & 0 & 10 \\
\hline Number of delivery & 2.51 & 1.7 & 0 & 8 \\
\hline Number of abortion & 0.25 & 0.54 & 0 & 8 \\
\hline Number of living children & 2.52 & 1.69 & 0 & 8 \\
\hline Number of boys & 1.28 & 0.99 & 0 & 4 \\
\hline Number of girls & 1.32 & 1.00 & 0 & 6 \\
\hline
\end{tabular}

best-fitting model was then given as the proportion of $\mathrm{A}, \mathrm{C}$ or E accordingly.

\section{Model Fitting for Quantitative Data}

The typical statistical approach employed is known as maximum likelihood estimation and explained comprehensively elsewhere ${ }^{7}$ MX $\quad$ interface (http://statgen.iop.kcl.ac.uk/bgim/ twinfit.html) was used to find the best fitting model. Two variances and covariance were found for MZ and DZ using SPSS, and 6 outputs were placed in twin univariate model fitting. The number of $\mathrm{DZ}$ and $\mathrm{MZ}$ twins and type I error rate of 0.05 (the probability of falsely rejecting a model) was entered in the model.

\section{RESULTS}

\section{Descriptive Analysis}

A simple description of quantitative variables studied as reproductive behaviour is shown in Table 1 . Our total sample was 192 females or 96 twin pairs inclusive of 108 identical twins (MZ, 54 twin pairs) and 84 non-identical twin pairs (DZ, 42 twin pairs). Age of marriage and age of first pregnancy ranges between 10-35 years and 11-32 years, respectively. Marital age in Malay culture occurs often starts from a very young age protected by Sharia law. There was one case who married at age of 10,5 cases at the age of 12,3 cases at the age of 13 and 7 cases at the age of 14 . DZ twins were more likely to marry at an earlier age compared with $\mathrm{MZ}(19.15 \pm 4.85$ vs. $21.60 \pm 4.51, \mathrm{p}=0.002)$. This could inflate the genetic component of the model described below.

\section{Genetic Analysis}

\section{A classical approach using Falconer's formula}

There was a significant correlation between twin 1 and twin 2 for both MZ and DZ twins for measured variables $(\mathrm{p}=0.001)$ (Table 2). The similarity between the behaviour of twin 1 and twin 2 for the age of pregnancy in the MZ type is much higher than that of the DZ, leading to a heritability value of 0.68 . Other variables, such as the number of living children and deliveries, have a low heritability (0.245), while genetic contribution was above $50 \%$ for a number for pregnancies and age of marriage (Table 2).

A significant correlation was found between twin 1 and twin 2 for both MZ and DZ twins in all the quantitative variables measured including the age of marriage, age of first pregnancy, number of pregnancies, number of spontaneous miscarriage, number of living children, number of boys and girls (See 95\%CI for all these variables in Table 2). Maximum likelihood analysis was performed for reproductive behaviour traits but was not presented as the DZ variances were either larger than the variances for MZ twins or the covariance of $\mathrm{DZ}$ twins was higher than that of the MZ twins. Covariance measures the strength of correlation between individuals (Twin 1 and 2) for every measured trait. If a trait is inherited, the MZ correlation will be high, and therefore, the covariance should be large. In these circumstances, however, the value for DZ correlation is low, and therefore, their covariance should be small. One of the criteria for running the maximum likelihood analysis by MX is having a smaller covariance for the DZ twins than that of the MZ twins. If this assumption is violated, then the model will not run. Moreover, the distribution of quantitative variables was not normal as the Kolmogorov smirnov normality test was statistically significant for all these variables ( $p=0.001$, data is not shown). Data was, therefore, categorized and analyzed as qualitative outcomes.

\section{Qualitative Variables}

The frequency distribution of some of the qualitative variables considered as reproductive behaviour is shown in Table 3.

\section{Probandwise Concordance Rate and Model Fitting}

All but 15 cases were under prenatal, and postnatal care and only eight subjects did not breastfeed their baby. There was not sufficient data on miscarriage among DZ twins. It was

Table 2. Analysis of genetic and environment variable for variables related to quantitative reproductive behavior

\begin{tabular}{cccccc}
\hline Variable & $\mathbf{r}_{\mathrm{MZ}}$ & $\mathbf{r}_{\mathrm{DZ}}$ & ${ }^{*}$ Genetic component & ${ }^{* *}$ Shared environment & Non shared environment \\
\hline Age of marriage $(\mathrm{Y})$ & 0.712 & 0.551 & 0.583 & 0.129 & 0.288 \\
\hline Age of first pregnancy $(\mathrm{Y})$ & 0.713 & 0.241 & 0.971 & $<0.1$ & $<0.1$ \\
\hline Number of pregnancies & 0.790 & 0.563 & 0.674 & 0.116 & 0.21 \\
\hline Number of deliveries & 0.712 & 0.684 & 0.237 & 0.146 & 0.288 \\
\hline Number of abortions & 0.270 & 0.208 & 0.124 & 0.475 & 0.288 \\
\hline Number of living children & 0.712 & 0.684 & 0.237 & - & 0.131 \\
\hline Number of boys & 0.549 & 0.171 & 0.869 & 0.064 & 0.560 \\
\hline Number of girls & 0.440 & 0.511 & 0.376 & \\
\hline
\end{tabular}

*Genetic component is estimated using this formula: $\mathrm{h}^{2}=2\left(\mathrm{r}_{\mathrm{MZ}}-\mathrm{r}_{\mathrm{DZ}}\right)$.

***Environment component is divided into two portions inclusive of "shared environment" or common environment and "non-shared environment" or individual environment. Shared and non-shared environment are estimated using the following formulas respectively: $\mathrm{C}^{2}=\left(\mathrm{r}_{\mathrm{MZ}}\right.$ $-\mathrm{h}), \mathrm{E}^{2}=1-\mathrm{r}_{\mathrm{MZ}}$ 
Table 3. Description of qualitative variables of reproductive behavior for both MZ and DZ twins ( $\mathrm{n}=192)$

\begin{tabular}{lcc}
\hline Variable & Frequency & Percentage \\
\hline Prenatal care during last pregnancy & 15 & 12.3 \\
$\quad$ Government Hospital & 75 & 61.5 \\
$\quad$ Government Clinic & 27 & 22.1 \\
$\quad$ Private hospital/clinic & 5 & 4.1 \\
$\quad$ None & 46 & 40 \\
\hline Postnatal care during last pregnancy & 55 & 47.8 \\
$\quad$ Government Hospital & 9 & 7.8 \\
$\quad$ Government Clinic & 5 & 4.3 \\
$\quad$ Private hospital/clinic & 117 & 93.6 \\
$\quad$ None & 128 & 48.1 \\
\hline Did you breastfeed your baby? & 128 & 55.2 \\
\hline Did you follow a family planning program? & 77 & 46.1 \\
\hline Have you done pap smear during last year? & 55 & 44.7 \\
\hline Age of marriage more than 22 years & 53 & 44.9 \\
\hline Age of first pregnancy more than 22 years & 43 & 37.1 \\
\hline More than 3 pregnancies & 100 & 85.5 \\
\hline More than 3 deliveries & 89 & 76.6 \\
\hline Any spontaneous miscarriage & 93 & 79.5 \\
\hline More than 3 children & 82 & 70.1 \\
\hline Having a boy &
\end{tabular}

therefore not possible to estimate the probandwise concordance rate for $\mathrm{MZ}$ and $\mathrm{DZ}$ twins for these measured variables of reproductive behaviour due to lack of numbers. Probandwise correlations for the following variables were higher than 1, suggesting genetic influence: family planning, pap smear, age of marriage (prior to 22 years), age of first pregnancy (before 22 years), and number of children, girls and boys (more than 3). Tetrachoric correlation for these data was estimated as shown in Table 4. Values were lower for DZ twins than MZ twins. Genetic modelling for these variables was performed, and it was found that for both all the variables CE (C91\%, E9\%) and (C85\%, E15\%) was the best-fitted model.

\section{DISCUSSION}

\section{Reproductive Behavior}

Genetic effects on fertility do not originate only through direct biological processes; in fact, the role of human traits and behaviors under volitional control is probably greater (Rodgers et al., 2001).

Measures of reproductive behavior such as the age of marriage, age of first pregnancy, number of pregnancies, and number of children were measured in our study using a basic approach. However, it was not possible to run a univariate twin analysis, as the covariance for DZ twins was found to be higher than that of the MZ twins, a finding that would make it impossible to run the matrix analysis. It is worth noting that the early age of marriage is correlated with the number of pregnancies, followed by other quantitative phenotypes representing reproductive behavior. These data was then categorized and analyzed as qualitative data.

Adopting family planning programs and taking pap smear tests were also asked about in our study as measures of reproductive health behavior, both of which were found to have higher proband-wise MZ to DZ concordance ratio more than 1 . The model fitting analysis suggested CE model as the best-fitted model for all the above-mentioned variables.

A major twin study on reproductive behavior was done by Fisher (1920). According to his fundamental theorem of natural selection, the size of the descendant pool deriving from "high reproducers" overwhelms the pool of descendants of "low reproducers". Genetic variance in human fertility was shown by Fisher to be around 0.40 among British aristocrats (for variable family size variable). Houle et al. (1920) later found a low heritability for father-child pairs (0.02) and mother-child pairs (0.12). International data from twins raised apart also showed a low heritability value for fertility $(0.12)$ (Mealey and Segal, 1993) Kohler et al. (Between Nurture and Nature: The Shifting Determinants of Female Fertility in Danish Twin Cohorts - Journals - NCBI, no date) looked at the heritability of family size among Danish twins and found a significant influence of genes. Greater similarity of the completed fertility among female monozygotic twins was found as compared to dizygotic twins, meaning that the genetic influence on the fertility of females increased over the sample period. Some other investigators studied the age of first intercourse (Miller et al., 1999), age of marriage (Fisher, 1920), age of first pregnancy (Fisher, 1920), and fertility expectations (Rodgers JL, Rowe DC) among twins to estimate the heritability rate. Kohler et al. (2002) found a high heritability for fertility in Denmark during the demographic transition where high levels of personal choices in fertility decision-making were available to Danish citizens. Rodgers et al. (n.d.) reported a heritability of 0.33 for fertility in a US survey, and moderate heritability for age at first pregnancy.

An Australian twin study found a significant overlapping genetic variance between the first age of reproduction, age of menopause and reproductive fitness measures (Kirk et al., 2001). Our study result is consistent with that of Rodgers et al. (2001), which reported a small but significant genetic variance for measured variables such as a number of children. However, our data for the age of pregnancy shows a higher heritability than Rodgers et al. study. This could be due to variation in social concepts of fertility, demographic variations between the countries, and freedom of choice for fertility.

Direct and involuntary biological processes, therefore, seem to be under genetic influence as adaptive processes. For instance, a mutation causing resistance to disease in utero would increase surviving family size, and therefore both phenotypic and genetic variance. Mutations can also change

Table 4. Probandwise concordance rate of MZ and DZ twins, its ratio and tetrachoric correlation for qualitative reproductive behavior

\begin{tabular}{|c|c|c|c|c|c|}
\hline Variable name & $\begin{array}{c}\text { Probandwise concordance } \\
\text { rate for MZ twins }\end{array}$ & $\begin{array}{c}\text { Probandwise concordance } \\
\text { rate for } \mathrm{DZ} \text { twins }\end{array}$ & Ratio & $\begin{array}{c}\text { Tetrachoric Correlation } \\
\text { rMZ }\end{array}$ & $\begin{array}{c}\text { Tetrachoric Correlation } \\
\text { rDZ }\end{array}$ \\
\hline Family planning & 0.888 & 0.733 & 1.21 & 0.92 & 0.85 \\
\hline Pap smear & 0.904 & 0.750 & 1.205 & 0.96 & 0.66 \\
\hline
\end{tabular}


genetic variance and contribute to reproductive success. Beauty or charisma, for example, cause sexual attraction. Another theory that can explain genetic variation is "gene flow" in partially isolated populations, over-dominance of surviving gene, as explained by Hughes (Rodgers and Rowe, n.d.). She shows empirical evidence that phenotypic variance for human fitness traits and fertility are likely due to genetic polymorphism that is actively maintained by natural selection. The remainder of phenotypic variance, she believes, is either non-genetic or due to non-adaptive forces such as mutation.

However, many disruptive factors in today's society contribute to maintaining genetic variance for fertility and reproduction success within the population. These factors are inclusive of changes in societal norms about sexual attraction, marriage and mating; access to contraceptive methods and availability of family planning choices; changes in desires and norms for family size; treatment of human infertility, laws and regulations regarding abortion; and the economic status of families. Fertility, therefore, is strongly behavioral as well, even though a biological component has been introduced. Rodgers et al. (2001) argue that fertility differentials are genetically influenced, and at least part of the influence derives from behavioral precursors that are under volitional control, which are themselves genetically mediated. The latter is explained by Miller et al. (2000), who suggested a theoretical framework relating motivation for having children to genetic determinants through neural substrates. Miller et al. divided motivations for having children into positive and negative. They then postulated that positive childbearing motivation originates from traits such as nurturance and affiliation and that negative childbearing motivation derives from securityrelated traits such as avoidance of harm and anxiety (over having children). According to Miller et al., these traits are under the influence of biochemical messengers secreted from neurotransmitters, which in turn are under the influence of genes. Other studies also show a relation between reproductive physiology and reproductive behavior. (Randolph, 2008; Stuckey, 2008). In these studies, reproductive behavior is measured by fertility outcomes, age of first intercourse, age of first pregnancy, number of living children, and so on. Substantial evidence suggests that these variables have a heritable component. There are different sources of genetic influence on fertility variance and other components of reproduction behavior, including gene-gene interaction or gene-environment interaction. Different processes of mutation, heterozygote advantage or overdominance (as in Fisher's model mentioned earlier), sexual antagonism and environmental perturbations re-introduce genetic variance into fitness traits even though the process of natural selection is washing it out.

Udry (1996) suggested that biologically based variance can only emerge in cultural settings where a substantial choice of fertility is available (Udry, 1996a). He, therefore, believed that a high level of heritability can only be observed in modern societies in which contraception is practiced and not in societies where many follow a natural fertility regimen, i.e., unprotected sex, justified by societal and biological norms to have children as often as possible, which will hide the individual differences in fertility motivation. Kohler et al.'s results with high heritability for fertility outcome support
Udry's theory, as their study was done during fertility transition (when falling family sizes reflected increased individual choices over reproduction). In order to test Udry's theory, a longitudinal study of generations of twins is recommended to investigate the heritability of fertility and reproductive behavior measures in a full reproductive life span. An epidemiological study to compare heritability of these measures among societies that practice contraception with those that do not can ultimately prove or reject his theory.

The biological, social and reproductive characteristics of women who have had twins were compared with those of other parous women using questionnaire data gathered for a prospective cohort study of women aged 35 and over on the island of Guernsey. Data for 97 mothers of twins and 4026 other parous women were available for analysis. This study did not provide evidence that mothers of twins differ markedly from other parous women across a range of characteristics that might be associated with twinning or associated with breast cancer risk (Murphy et al., 1998). A more recent study investigated the genetic behavior of 4925 Finnish twins aged 23-27 years old (Mustanski et al., 2007). Investigators evaluated theoretical perspectives that differentially emphasize biological dispositions, social/cultural factors, or universal pathways to explain individual differences in sexual behaviors using a twin study design. The following variables were studied: self-report information on initiation/abstinence of sexual intercourse, onset age, and number of sexual partners. There were trends for common environmental influences on the initiation of sexual activity and, in females, age at first intercourse. Some differential effects in males and females were found. Results comparing "onset age of sexual activity" and "number of partners" among experienced twins from pairs concordant and discordant for initiation found genetic and environmental influences on initiation/abstinence overlapped those found for the other aspects of sexual behavior.

\section{Study Limitations}

The following limitations may apply to this particular research; suggested solutions are discussed in the parentheses.

- One twin might be unwilling to participate in the study. This may lead to loss of data on both twins (interviewers tried to encourage participation via the twin brother or sister). Eight percent of cases among our twins did not wish to be interviewed for this study.

- Twins were recruited using media-based advertising. Those who did not use radio could have been left out of the study. For this reason, pamphlets were distributed in various public places such as schools, colleges, and universities. This could target a group of youngsters and educated twins with higher class in society, leading them to register more. Thus, it was decided that other public places such as shopping centers, mosques, and hospitals should also be considered for advertising and distribution of pamphlets and posters. This study is volunteer-based, and it is well known to the researchers that those who did not volunteer could be potentially left out. To solve this problem, we used the snowball technique, meaning that 
the research assistants asked the subjects to tell other twins about the study and encourage them to participate.

- Was there any need for comparing the twin results with that of controls drawn from the normal population? The nature of a twin study is based on a comparison between twins of different sorts (MZ and DZ). Any variation between the twins for the measured variable is considered using within-twin and between-twin differences. Therefore, the results of this study are only applicable to twins, and this makes a comparison with the normal population irrelevant.

- Could combining data from two different races (Malay, Chinese, Indian) affect the result? Multiracial studies are common for twin analysis when the sample size is not adequate, or the proband is hard to identify. For instance, Glinianaia et al. (2006) studied twin data for cerebral palsy from 9 centers in Europe. Behavioral scientists have also used cross-cultural studies of twins, pooling all the data together and estimating the heritability values (Udry, 1996b). A mixture of twins from different ethnicities (85\% European American, 13.4\% African American, and 1\% Hispanic, Asian or Native American) from Missouri twin samples and samples from Dutch twin registries were combined for analysis. Moskowitz (1999) (Hudziak et al., 2004) and Schieken (1998) (Udry, 1996b) studied passive childhood smoking and its relation with coronary artery disease risk among twins, compiling data from various races ( 85 black and 323 whites). It is therefore concluded that collapsing data from various races or cultures should not interfere with the heritability of a trait, although the effect of the environment might be different. This environmental difference is still shared by members of a twin pair (MZ and DZ) for common environmental factors. The individual variation between twins in each twin set is expected for non-shared environmental effects and could be affected by cultural differences, but it still defines a variation between twins within each pair.

- According to Bruder et al. (Bruder et al., 2008), identical twin studies may not be $100 \%$ similar. The presumption has always been that identical twins are identical down to their DNA. His finding suggests that there are small, subtle differences due to copy number variation. Those differences may point the way to a better understanding of genetic diseases when we study discordant monozygotic twins where one twin has a disorder, and the other does not.

In conclusion, these results documented genetic variation in individual differences in the sexual behavior of young adults. Genetic dispositions should be considered as an important contributor in the integrated models of twins' sexual behavior to facilitate more effective health promotion and risk-taking intervention.

\section{REFERENCES}

Between Nurture and Nature: The Shifting Determinants of Female Fertility in Danish Twin Cohorts - Journals - NCBI (n.d.). Available at: https://www.ncbi.nlm.nih.gov/labs/ articles/14652919/ (Accessed: 28 June 2017).
Bruder, C. E. G., et al. (2008). Phenotypically Concordant and Discordant Monozygotic Twins Display Different DNA Copy-Number-Variation Profiles. American Journal of Human Genetics, 82(3), 763-771. https://doi.org/10.1016/ j.ajhg.2007.12.011

Christensen, K., et al. (2003). The correlation of fecundability among twins: evidence of a genetic effect on fertility? Epidemiology (Cambridge, Mass.). United States, 14(1), 6064. https://doi.org/10.1097/01.EDE.0000039182.92283.E1

Day, F. R., et al. (2015). Causal mechanisms and balancing selection inferred from genetic associations with polycystic ovary syndrome. Nature communications. England, 6, 8464. https://doi.org/10.1038/ncomms9464

Fisher, R. A. (1920). The genetical theory of natural selection. Oxford, England: Clarendon Press.

Glinianaia, S. V., et al. (2006). Intrauterine growth and cerebral palsy in twins: a European multicenter study. Twin research and human genetics: the official journal of the International Society for Twin Studies. England, 9(3), 460466. https://doi.org/10.1375/183242706777591209

Houle D. (1992) 'Comparing evolvability and variability of quantitative traits', Genetics, 130, pp. 195-204.

Hudziak, J. J., et al. (2004). Genetic and environmental contributions to the Child Behavior Checklist ObsessiveCompulsive Scale: a cross-cultural twin study. Archives of general psychiatry. United States, 61(6), 608-616. https://doi.org/10.1001/archpsyc.61.6.608

Jahanfar, S. (2008). National Malaysian twin registry - A perfect opportunity for researchers to study nature versus nurture. Malaysian Family Physician, 3(2), 111-112.

Jahanfar, S. and Jaffar, S. H. (2013). Malaysian Twin Registry. Twin Research and Human Genetics, 16(01), 246-247. https://doi.org/10.1017/thg.2012.80

Jahanfar, S., Lye, M.-S. and Krishnarajah, I. S. (2013). Genetic and environmental effects on age at menarche, and its relationship with reproductive health in twins. Indian journal of human genetics. Medknow Publications, 19(2), 245-250. https://doi.org/10.4103/0971-6866.116127

Kendler, K. S., et al. (1993). A test of the equal-environment assumption in twin studies of psychiatric illness. Behavior Genetics, 23(1), 21-27. https://doi.org/10.1007/BF01067551

Kirk, K. M., et al. (2001). Natural selection and quantitative genetics of life-history traits in Western women: a twin study. Evolution; international journal of organic evolution. United States, 55(2), 423-435.

Kohler, H. P., et al. (2002). The fertility pattern of twins and the general population compared: Evidence from Danish cohorts 1945-64. Demographic Research, 6, 383-408. https://doi.org/10.4054/DemRes.2002.6.14

Mealey, L. and Segal, N. L. (1993). Heritable and environmental variables affect reproduction-related behaviors, but not ultimate reproductive success. Personality and Individual Differences, 14(6), 783-794. https://doi.org/10.1016/01918869(93)90091-G 
Mehta, D., et al. (2016). Evidence for Genetic Overlap Between Schizophrenia and Age at First Birth in Women. JAMA psychiatry. United States, 73(5), 497-505. https://doi.org/10.1001/jamapsychiatry.2016.0129

Miller, W. B., Pasta, D., MacMurray, J. and Muhleman, D. C. D. (2000). Genetic influence on childbearing motivation: Further testing a theoretical framework. In D. C. Rodgers and M. W. E. Rowe (ed.) Genetic influences on human fertility and sexuality (pp. 67-84). Boston: Kluwer Academic Publishers. https://doi.org/10.1007/978-1-4615-4467-8_4

Murphy, M., et al. (1998). Social, biological and reproductive characteristics of mothers of twins: implications for breast cancer risk. Annals of human biology. M. Murphy, Imperial Cancer Research Fund, General Practice Research Group, University of Oxford, UK., 25(1), 77-85. https://doi.org/ 10.1080/03014469800005442

Mustanski, B., et al. (2007). Sexual behavior in young adulthood: A population-based twin study. Health Psychology. Rose, Richard J.: Department of Psychological and Brain Sciences, 1101 East Tenth Street, Bloomington, IN, US, 47405, rose@indiana.edu: American Psychological Association, 26(5), 610-617. https://doi.org/10.1037/02786133.26.5.610

Neale, M. C., et al. (1997). Model fitting functions and optimization methodology for genetic studies of twins and families.
Rahmioglu, N., et al. (2014). Genetic variants underlying risk of endometriosis: insights from meta-analysis of eight genome-wide association and replication datasets. Human reproduction update. England, 20(5), 702-716. https://doi.org/10.1093/humupd/dmu015

Randolph, J. F. (2008). The endocrinology of the reproductive years. Journal of Sexual Medicine, 5(10), 2274-2281. https://doi.org/10.1111/j.1743-6109.2008.00919.x

Rodgers, J. L., et al. (2001). Variation in Human Fertility: Evidence from Recent Behavioral and Molecular Genetic Studies, 10(5), 184-188. https://doi.org/10.1111/14678721.00145

Rodgers, J. L., Rowe, D. C., et al. (eds) (n.d.). Evolutionary causes and consequence of variation in fertility and other fitness traits. In Genetic influences of human fertility and sexuality (pp. 7-33). Boston: Kluwer.

Stuckey, B. G. A. (2008). Female sexual function and dysfunction in the reproductive years: the influence of endogenous and exogenous sex hormones. The journal of sexual medicine. Netherlands, 5(10), 2282-2290. https://doi.org/10.1111/j.1743-6109.2008.00992.x

Udry, J. (1996a). Biosocial models of low-fertility societies. In E. J. B. Casterline, R. D. Lee and K. A. Foote (Ed.), Population and Development Review (pp. 325-336). New York: The Population Council, 22(Suppl). https://doi.org/10.2307/ 2808017

Udry, J. (1996b). Biosocial models of low-fertility societies. E. J. B. Casterline, R. D. Lee and K. A. Foote (Ed.). New York: The Population Council, p. 96. 